\title{
From 1972 to GSAS-II
}

Robert Von Dreele ${ }^{a}$

${ }^{a}$ Advanced Photon Source, Argonne National Laboratory, Lemont, IL 60439, USA, vondreele@anl.gov

In 1972 while I was a post doc working in J.S. Anderson's laboratory at Oxford University, Tony Cheetham, doing a postdoc with Brian Fender, stopped by and asked if I would be interested in doing a neutron powder diffraction experiment. We obtained from Alan Hewat a copy of a program written by Hugo Rietveld for the analysis. It was too small (array sizes, mostly) to work on our problems (Ti-niobium oxides) and making the arrays large enough made it too big for the Oxford computer center machine (an ICL 1906a), so I had to extensively modify it so it could analyze these large structures. Thus began my travels in crystallographic computing as a means of doing my science. In this talk, I'll cover further developments of what we now know as the Rietveld Method and how that lead in turn to analysis of $x$ ray data, neutron TOF data, the creation of GSAS and now GSAS-II.

Acknowledgement: This work was supported by the US DOE under contract Nos. W-7405-ENG36 (Los Alamos) and DE-ACO2-06CH11357 (Argonne). 